\title{
Medo e ansiedade dos estudantes de diferentes classes sociais ao tratamento odontológico
}

\author{
Fear and anxiety of students of different social classes \\ when facing dental treatment \\ El miedo y la ansiedad de los estudiantes de diferentes clases sociales \\ a tratamiento dental \\ Ronald Jefferson MARTINS ${ }^{1}$ \\ Naiana de Melo BELILA ${ }^{2}$ \\ Cléa Adas Saliba GARBIN ${ }^{1}$ \\ Artênio José Ísper GARBIN ${ }^{\mathbf{1}}$ \\ ${ }^{1}$ Postgraduate Program in Social and Preventive Dentistry, Faculty of Dentistry of Araçatuba, \\ UNESP - Univ Estadual Paulista, Araçatuba, São Paulo, Brazil \\ ${ }^{2}$ Professor Graduate Program in Social and Preventive Dentistry, Faculty of Dentistry of Araçatuba, \\ UNESP - Univ Estadual Paulista, Araçatuba, São Paulo, Brazil
}

\begin{abstract}
Resumo
Introdução: A expectativa de dor frente ao tratamento dentário se perpetuou durante séculos, gerando medo e ansiedade. Objetivo: $O$ objetivo do trabalho foi verificar a associação do medo e ansiedade ao tratamento odontológico com a classe socioeconômica de adolescentes do ensino fundamental. Metodologia: Participaram desta pesquisa os adolescentes regularmente matriculados no ensino fundamental das escolas públicas de dois municípios do noroeste do Estado de São Paulo, Brasil. Aplicou-se o questionário "Instrumental de Avaliação Socioeconômica", a fim de classificar as famílias quanto à classe socioeconômica e o "Dental FearSurvey (DFS)", para verificar o medo e ansiedade frente ao tratamento odontológico. Resultados: Participaram 179 (43,3\%) adolescentes, desses a maioria pertencia à classe Baixa Superior (61\%). Houve relação significativa entre subgrupos da escala DFS e classes socioeconômicas. Conclusão: Existe associação entre o nível de medo e ansiedade ao tratamento odontológico e a classe socioeconômica dos estudantes.

Descritores: Medo; Ansiedade; Assistência Odontológica.
\end{abstract}

\begin{abstract}
Introduction: The expectation of pain during dental treatment has perpetuated for centuries, generating fear and anxiety. Objective: The aim of the study was to verify the association of dental fear and anxiety and socioeconomic class of fundamental schooling teenagers. Methods: Teenagers regularly enrolled in fundamental schooling at public schools of two towns in the northwestern region of the State of São Paulo, Brazil, took part in the study. The questionnaire "Socioeconomic Assessment Tool" was used to classify the families with regards to their socioeconomic class and the "Dental Fear Survey (DFS)" was used to verify fear and anxiety towards dental treatment. Results: 179 (43.3\%) teenagers participated in the study, most belonged to the Upper Lower class (61\%). There was a significant relationship between DFS scale subgroups and socioeconomic classes. Conclusion: There is a significant relationship between the participants' level of fear and anxiety towards dental treatment and the different socioeconomic classes.

Descriptors: Fear; Anxiety; Dental Care.

\section{Resumen}

Introduccíon: La expectativa de dolor durante el tratamiento dental se ha perpetuado durante siglos, generando miedo y ansiedad. Objetivo: El objetivo fue verificar la asociación de miedo y ansiedad al tratamiento dental para los adolescentes de clase socioeconómica de la escuela primaria. Métodos: Se analizaron los adolescentes matriculados en la enseñanza primaria en las escuelas públicas en dos condados del noroeste del estado de Sao Paulo, Brasil. Se utilizó el cuestionario "Instrumental evaluación socioeconómica" con el fin de clasificar los hogares como a la clase socioeconómica y el "Dental FearSurvey (DFS)", para comprobar el miedo y la ansiedad durante el tratamiento dental. Resultados: 179 participantes $(43,3 \%)$ de los adolescentes, la mayoría de ellos pertenecían a la clase baja-alta $(61 \%)$. Se observó una relación significativa entre los subgrupos de escala DFS y clases socioeconómicas. Conclusión: Existe una asociación entre el nivel de miedo y ansiedad al tratamiento dental y el nivel socioeconómico de los estudiantes.
\end{abstract}

Descriptores: Miedo; Ansiedad; Cuidado Dental.

\section{INTRODUÇÃO}

A expectativa de dor frente ao tratamento dentário se perpetuou durante séculos, gerando medo e ansiedade ${ }^{1}$. Apesar do progresso da odontologia no controle da dor em todo o mundo, hodiernamente persiste o estereótipo negativo do cirurgião-dentista em páginas da web e produções cinematográficas $^{2,3,4}$.

O medo pode ser definido como o temor a algo ou alguma coisa que represente um perigo real, como ameaça a integridade física ou psicológica da pessoa, o que leva a um estado emocional de alerta ante ao perigo. Já a ansiedade é um temor sem um objeto real, causada por lembranças e experiências anteriores, estando relacionados a uma etiologia multifatorial. É influenciada por aspectos internos do indivíduo, o ambiente em que ele vive e as condições do atendimento que é submetido 5 .

Esses sentimentos frente ao tratamento odontológico revelam-se por comportamentos arredios e alguns sinais; como por exemplo, queixa verbal, inquietação, palidez da pele, midríase, transpiração excessiva, formigamento das extremidades, aumento da pressão arterial e taquicardia; entre outros. A intensidade desses sentimentos varia de um paciente para outro; ou no próprio paciente, dependendo do 
tipo de procedimento que irá ser submetido, mostrando-se como uma barreira para um atendimento mais eficiente pelo cirurgião-dentista ${ }^{5}$.

A ansiedade apresenta grande impacto no aspecto psicossocial do indivíduo, pois a maioria dos pacientes que evitam o tratamento odontológico por muito tempo acaba por ter péssimas condições de saúde bucal com a perda de dentes, sentindo vergonha pela falta dos elementos dentários, dificuldades de se relacionar com outras pessoas e ingressar no mercado de trabalho ${ }^{6}$.

Em especial, as famílias com pior nível de renda, escolaridade, cultural e perfil demográfico; além de menor acesso as ações e atividades educativas, preventivas e curativas estão associadas com a alta prevalência de experiências desagradáveis causadas por doenças bucais; como a cárie severa que acomete a cavidade bucal de crianças e adolescentes, gerando um impacto extremamente negativo na qualidade de vida desses indivíduos ${ }^{7}$.

Poucas pesquisas procuraram avaliar o nível de medo e ansiedade frente ao atendimento odontológico em indivíduos de diferentes níveis socioeconômicos. Baseado no que foi descrito, o trabalho teve como objetivo verificar a associação entre medo e ansiedade ao tratamento odontológico e a classe socioeconômica de adolescentes do ensino fundamental de dois municípios da região noroeste do Estado de São Paulo, Brasil.

\section{MATERIAL E MÉTODO}

O estudo consiste em uma pesquisa descritiva, com caráter transversal e abordagem quantitativa, realizada no período de julho a setembro de 2015, nas escolas de ensino fundamental de dois municípios de pequeno porte da região noroeste paulista: Américo de Campos e Pontes Gestal. Esses municípios foram escolhidos por apresentarem pequeno porte, população predominantemente urbana e Índice de Desenvolvimento Humano (IDH-M), de renda (IDH-M Renda) e de educação (IDH-M Educação) semelhantes.

Inicialmente, contataram-se os secretários municipais de educação e diretores das escolas para esclarecimento do propósito da pesquisa e posterior uso dos dados coletados, com a finalidade de obter apoio para realização do trabalho. Os mesmos esclarecimentos foram dados aos pais ou responsáveis pelos alunos no dia da reunião de pais e mestres.

Participaram desta pesquisa todos os estudantes de 11 a 14 anos, regularmente matriculados no ensino fundamental das escolas públicas dos municípios. Foram excluídos os que devolveram o questionário respondido de forma incompleta, ou aqueles cujos pais ou responsáveis não assinaram o termo de consentimento livre e esclarecido.

Os pais ou responsáveis pelos estudantes responderam ao questionário:

$>$ Instrumental de Avaliação Socioeconômica, a fim de classificar as famílias quanto à classe socioeconômica. Nesta escala, a situação econômica da família vale de 01 a 21 pontos (renda bruta de até $1 / 2$ salário mínimo a mais de 100 salários mínimos), o número de membros residentes vale de 01 a 06 pontos (acima de 8 até 1 a 2 membros), escolaridade dos familiares vale de 0 a 07 pontos (analfabeto até superior completo), condição / situação da habitação vale de 0 a 10 pontos e ocupação do chefe da família vale de 01 a 13 pontos. A soma desses indicadores classifica as famílias em seis classes, classe Baixa Inferior (BI), Baixa Superior (BS), Média Inferior (MI), Média (ME), Média Superior (MS) e Alta (AL) sendo a "Alta" (de 55 a 57 pontos) a mais favorecida e a "Baixa Inferior" (de 0 a 20 pontos) a menos favorecida ${ }^{8}$.

Já os estudantes responderam ao questionário:

> Dental FearSurvey (DFS), a fim de verificar o nível de medo e ansiedade do paciente frente ao atendimento odontológico. Desenvolvido originalmente por Kleinknechtet et al. ${ }^{9}$ e validado no Brasil por César et al. ${ }^{10}$, sendo um instrumento de autoavaliação que analisa os motivos pelo qual os indivíduos evitam a procura da consulta e seus sintomas fisiológicos durante a realização do tratamento odontológico. A pontuação varia de 20 a 100 , sendo que "20 pontos" correspondem à ansiedade baixa e "100 pontos" a ansiedade elevada. São avaliadas 20 situações distintas relacionadas com a consulta ao cirurgiãodentista: os itens 1 e 2 referem-se ao evitamento da consulta; 3 a 7 correspondem a sinais auto-percepcionados de ativação fisiológica durante a consulta; 8 a 19 equivalem a estímulos e procedimentos específicos em que é avaliado o medo, ansiedade ou desconforto; e o item 20 faculta uma classificação global do medo perante a consulta ao cirurgiãodentista. Todos os itens são classificados com respostas em uma escala tipo Likert, que varia de forma crescente de 1 a 5 pontos. Nos itens de 1 a7 a classificação varia de "1" para "nunca" a " 5 " para "quase sempre". Nos itens de 8 a20, a classificação varia de "1" para "nenhum medo" a " 5 " para "muito medo". Esta escala é dividida em três subgrupos de respostas: "Evitamento da consulta", sendo composto pelas questões 1, 2 e 8 a 13; "Ativação fisiológica durante a consulta" contendo as questões de 3 a 7; e "Medo, ansiedade ou desconforto relacionados com estímulos e procedimentos específicos", incluindo as questões de 14 a 20. Esta escala permite analisar item a item, de forma a detectar o medo induzido individualmente por cada estimulo ${ }^{11}$.

Os dados dos questionários foram tabulados em planilhas do programa Excel e analisados através do programa BioStat $^{12}$. Devido às pontuações não serem distribuídas normalmente, as variáveis foram analisadas através do teste Kruskal-Wallis não paramétrico, a fim de avaliar a diferença de escores médios entre os grupos de diferentes classes socioeconômicas, com nível de significância de $\mathrm{p}<0,05$.

A pesquisa foi aprovada pelo Comitê de Ética em Pesquisa em Seres Humanos, dentro dos padrões exigidos pela Resolução 466/12, com o número de processo CAAE: 39094214.2.0000.5420.

\section{RESULTADOS}

O universo amostral foi constituído por 412 pais e seus filhos, obtendo uma amostra de 179 respondentes $(43,4 \%)$. Pelo cálculo de amostra com erro amostral de $7 \%$ e nível de confiança de $95 \%$, a amostra necessária seria de 134 indivíduos, tendo sido obtido uma porcentagem de $22,1 \%$ a mais no tamanho da amostra.

Baseado no Instrumental de Avaliação Socioeconômica, $20,4 \%$ dos chefes da família pertenciam a Classe BI, $61 \%$ a Classe BS, $14,5 \%$ a Classe MI e $4,1 \%$ a Classe ME. Devido ao fato de apenas um único participante pertencer a Classe MS, este foi incluído na classe ME.

Em relação às questões de 1 a 7 (sentimentos em relação ao tratamento), as respostas "frequentemente" e "quase sempre" apareceram apenas nas classes BI e BS, com frequência de $6,2 \%$. Já nas questões de 8 a 20 (intensidade do medo), as respostas "bastante" e "muito" possuíram maior aparição nas classes BI e BS, com índice de 17,3\% (Tabelas 1 e 2).

Utilizou-se o teste não paramétrico de Kruskal Wallis para avaliar a diferença de escores médios entre os grupos 
das classes econômicas, com o nível de significância de 5\%. Por ter sido detectada diferença significativa entre as classes socioeconômicas $(p<0,01)$, a análise foi continuada com o Teste Dunn de comparações múltiplas entre os subgrupos "Evitamento da consulta" nas classes BI x MI e BS x MI, "Ativação fisiológica durante a consulta" nas classes
BI x MI e BS x MI e "Medo, ansiedade ou desconforto relacionados com estímulos e procedimentos específicos" nas classes BI x MI, BI x ME, BS x MI e BS x ME. Nos escores totais dos subgrupos, também houve relação significativa entre $\mathrm{BI} \times \mathrm{MI}, \mathrm{BI} \times \mathrm{ME}, \mathrm{BS} \times \mathrm{MI}$ e BS x ME (Tabela 3).

Tabela 1. Distribuição das respostas do questionário DFS (questões de 1 a 7) segundo as classes socioeconômicas, Araçatuba, 2015

\begin{tabular}{|c|c|c|c|c|c|c|c|c|c|c|c|c|}
\hline \multicolumn{13}{|c|}{ DFS } \\
\hline \multicolumn{2}{|l|}{ Respostas } & \multicolumn{2}{|c|}{ Nunca } & \multicolumn{2}{|c|}{$\begin{array}{c}\text { Uma ou duas } \\
\text { vezes }\end{array}$} & \multicolumn{2}{|c|}{ Algumas vezes } & \multicolumn{2}{|c|}{$\begin{array}{l}\text { Com } \\
\text { frequência }\end{array}$} & \multicolumn{2}{|c|}{ Quase sempre } & \multirow{2}{*}{$\begin{array}{c}\text { Tota } \\
\%\end{array}$} \\
\hline $\begin{array}{c}\text { Classe } \\
\text { Socioeconômica }\end{array}$ & $\mathrm{n}$ & $\%$ & $\mathrm{n}$ & $\%$ & $\mathrm{n}$ & $\%$ & $\mathrm{n}$ & $\%$ & $n$ & $\%$ & $\mathrm{n}$ & \\
\hline BI & 163 & 13,2 & 43 & 3,5 & 40 & 3,2 & 16 & 1,2 & 4 & 0,2 & 266 & 21,3 \\
\hline BS & 449 & 36,2 & 117 & 9,4 & 130 & 10,5 & 30 & 2,4 & 30 & 2,4 & 756 & 60,9 \\
\hline MI & 153 & 12,3 & 17 & 1,3 & 6 & 0,4 & - & - & - & - & 176 & 14 \\
\hline ME & 37 & 3 & 5 & 0,3 & 7 & 0,5 & - & - & - & - & 49 & 3.8 \\
\hline Total & 802 & 64,7 & 182 & 14,5 & 183 & 14,6 & 46 & 3,6 & 34 & 2,6 & 1237 & 100 \\
\hline
\end{tabular}

Tabela 2. Distribuição das respostas do questionário DFS (questões de 8 a 20) segundo as classes socioeconômicas, Araçatuba, 2015

\begin{tabular}{|c|c|c|c|c|c|c|c|c|c|c|c|c|}
\hline \multirow{2}{*}{$\begin{array}{c}\text { Respostas } \\
\text { Classe } \\
\text { Socioeconômica }\end{array}$} & \multicolumn{2}{|c|}{ Nenhum } & \multicolumn{2}{|c|}{ Um pouco } & \multicolumn{2}{|c|}{$\begin{array}{l}\text { DFS } \\
\text { Algum }\end{array}$} & \multicolumn{2}{|c|}{ Bastante } & \multicolumn{2}{|c|}{ Muito } & \multicolumn{2}{|c|}{ Total } \\
\hline & $\mathrm{n}$ & $\%$ & $\mathrm{n}$ & $\%$ & $\mathrm{n}$ & $\%$ & $n$ & $\%$ & $n$ & $\%$ & $\mathrm{n}$ & $\%$ \\
\hline BI & 170 & 7,3 & 156 & 6,7 & 61 & 2,6 & 65 & 2,8 & 43 & 1,9 & 495 & 21,3 \\
\hline BS & 513 & 22,1 & 440 & 19 & 159 & 6,9 & 160 & 6,9 & 132 & 5,7 & 1.404 & 60,6 \\
\hline MI & 254 & 11 & 47 & 2 & 12 & 0,5 & 6 & 0,3 & 6 & 0,3 & 325 & 14,1 \\
\hline ME & 75 & 3,3 & 13 & 0,6 & 2 & 0,2 & 1 & 0,1 & - & - & 91 & 4 \\
\hline Total & 1.012 & 43,7 & 656 & 28,3 & 234 & 10,1 & 232 & 10 & 181 & 7,9 & 2.315 & 100 \\
\hline
\end{tabular}

Tabela 3. Associação entre os subgrupos da escala DFS e as classes socioeconômicas, Araçatuba, 2015

\begin{tabular}{|c|c|c|c|c|}
\hline \multicolumn{5}{|c|}{ DFS } \\
\hline Subgrupos & $\begin{array}{c}\text { Evitamento da } \\
\text { consulta }\end{array}$ & $\begin{array}{l}\text { Ativação } \\
\text { fisiológica }\end{array}$ & $\begin{array}{c}\text { Medo } \\
\text { específico }\end{array}$ & Total \\
\hline Classe & & Baixa Inferior & & \\
\hline Baixa Superior & ns & ns & ns & ns \\
\hline Média Inferior & $p<0,05$ & $p<0,05$ & $p<0,05$ & $p<0,05$ \\
\hline Média & ns & ns & $p<0,05$ & $p<0,05$ \\
\hline Subgrupos & $\begin{array}{c}\text { Evitamento da } \\
\text { consulta }\end{array}$ & $\begin{array}{l}\text { Ativação } \\
\text { fisiológica }\end{array}$ & $\begin{array}{c}\text { Medo } \\
\text { específico }\end{array}$ & Total \\
\hline Classe & & Baixa Superior & & \\
\hline Média Inferior & $p<0,05$ & $p<0,05$ & $p<0,05$ & $p<0,05$ \\
\hline Média & ns & ns & $p<0,05$ & $p<0,05$ \\
\hline Subgrupos & $\begin{array}{c}\text { Evitamento da } \\
\text { consulta }\end{array}$ & $\begin{array}{l}\text { Ativação } \\
\text { fisiológica }\end{array}$ & $\begin{array}{c}\text { Medo } \\
\text { específico }\end{array}$ & Total \\
\hline Classe & & Média Inferior & & \\
\hline Média & ns & ns & ns & ns \\
\hline
\end{tabular}

*Teste: Kruskal Wallis

\section{DISCUSSÃO}

Apesar de todos os avanços tecnológicos na odontologia, a ansiedade e o medo são comumente vistos como grandes obstáculos para a procura da assistência odontológica devido estarem ligados à possibilidade da ocorrência de dor e sofrimento ${ }^{13,14}$.

Esses sentimentos são características biológicas do ser humano, que antecedem a expectativa por momentos de perigo e tensão nunca vivenciados, ou experiências prévias ruins. Estudos vêm sendo realizados para verificar o grau de medo e ansiedade ao tratamento odontológico e estratégias para minimizar seus impactos sobre a saúde bucal ${ }^{14,15}$, devido ao longo do tempo ter sido passado a distintas populações relatos de experiências negativas, mitos e crenças a este tratamento.

No presente estudo, observou-se a presença de medo e ansiedade ao tratamento odontológico na maior parte dos pesquisados pertencentes às classes socioeconômicas baixas, corroborando uma pesquisa realizada nos centros 
universitários de um município do Estado de Sergipe, Brasil, onde se verificou que a probabilidade de um paciente apresentar medo e ansiedade ao tratamento dentário, aumenta progressivamente quando o indivíduo possui menor escolaridade, baixa renda familiar, falta de acesso à internet ou a jornais, baixa frequência de higiene bucal e naqueles que apenas buscam o atendimento somente no caso de odontalgias ou tratamentos curativos ${ }^{3}$.

Em contrapartida, um estudo realizado na Suécia não mostrou relação significativa entre escolaridade e renda familiar com o medo e a ansiedade ao tratamento odontológico, mas evidenciou o envolvimento com a cultura da população estudada ${ }^{16}$.

As longas jornadas de trabalho, incompatibilidade de horários, falta de acesso ao serviço público de saúde, ausência de profissionais nas escolas e de dinheiro para a procura do atendimento particular faz com que os indivíduos das classes socioeconômicas menos favorecidas procurem o atendimento odontológico apenas em situações extremas de dor $^{17}$.

A adesão tardia ao tratamento leva a evolução da gravidade dos problemas bucais e isso exigirá procedimentos complexos e mais invasivos, aumentando a probabilidade de experiências ruins e desconfortos, além de maiores custos financeiros; o que faz com que seja evitada a procura pelo atendimento odontológico devido ao medo de sentir dor ${ }^{14}$.

Em um estudo realizado no Reino Unido grande parte dos entrevistados relatou frequentes episódios de dor por razões odontológicas, todos pertencentes a classes socioeconômicas menos favorecidas ${ }^{18}$.

Essa observação também foi mostrada em uma pesquisa desenvolvida em um distrito industrial de Minas Gerais, Brasil, onde os indivíduos com menor escolaridade e renda revelaram maior frequência de dor de origem dentária ${ }^{19}$.

Por outro lado, a ausência de odontalgia faz com que os indivíduos não considerem o tratamento odontológico como prioridade, não valorizando a saúde bucal e apresentando desinteresse pela sua própria saúde e de seus familiares, motivo que os leva a não retornarem na consulta após ter sido resolvida a dor ${ }^{20,21}$.

Esses achados fundamentam nosso trabalho, que revelou associação significativa entre os subgrupos da escala DFS; "Evitamento da consulta", "Ativação fisiológica durante a consulta" e no "Medo, ansiedade ou desconforto relacionados com estímulos e procedimentos específicos"; além do escore total com as classes socioeconômica baixa e média, como consequência das experiências anteriores ruins frente ao tratamento odontológico.

Os pacientes temem os procedimentos que serão submetidos dentro do consultório odontológico devido a experiências desagradáveis que tiveram, ou a relatos de pessoas próximas ao seu convívio, desconhecendo muitas vezes os recursos atuais para minimizar o estresse e a dor durante os procedimentos. Por sua vez, o adiamento da procura pelo tratamento faz com que haja necessidade da realização de procedimentos mais complexos, passíveis da ocorrência de dor. Cabe aos profissionais da área odontológica estabelecer maior vínculo de confiança e melhor relacionamento com seus pacientes, favorecendo assim a redução do medo e da ansiedade, e consequentemente diminuindo os impactos causados por estes sentimentos nas diversas populações ${ }^{14}$.

O processo de desconstrução do estereótipo negativo do cirurgião-dentista, historicamente formado, deverá necessariamente envolver nos tempos contemporâneos, saberes diversos que compõem a cultura midiática, erudita e popular $^{22}$.

\section{CONCLUSÃO}

O presente estudo sugere que existe associação entre as classes socioeconômicas e o medo e ansiedade frente ao tratamento odontológico. No entanto, novos estudos devem ser realizados a fim de se confirmar esta associação.

\section{REFERÊNCIAS}

1. Cruz JS, Cota LOM, Paixão HH, Pordeus IA. A imagem do cirurgião-dentista: um estudo de representação social. Rev Odontol Univ São Paulo. 1997; 11(4):307-13.

2. Pinho CB, Dias HS, Carvalho ACR, Barros SG Representação social da odontologia: a contribuição da produção cinematográfica para perpetuação de um estereótipo negativo. Rev Odontol Unesp. 2008; 37(3):275-81.

3. Carvalho RWF, Falcão PGCB, Campos GJL, Bastos AS, Pereira JC, Pereira MAS, Cardoso MSO, Vasconcelos BCE. Ansiedade frente ao tratamento odontológico: prevalência e fatores predictores em brasileiros. Ciênc saúde coletiva. 2012; 17(7):1915-22.

4. Nunes MF, Ferreira Netto AA, Silva PFP, Nogueira TE, Nunes VN. Imagem do cirurgião-dentista em sites de busca da web. Rev Eletr Enferm. 2012; 14(2):313-21.

5. Medeiros LA, Ramiro FMS, Lima CAA, Souza LMA, Fortes TMV, Groppo FC. Avaliação do grau de ansiedade dos pacientes antes de cirurgias orais menores. Rev Odontol UNESP. 2013; 42(5):357-63.

6. Vermaire JH, De Jongh A, Aartman IH. Dental anxiety and quality of life: the effect of dental treatment Community Dent Oral Epidemiol. 2008; 36(5):409-16.

7. Paredes SO, Galvão RN, Fonseca FRA. Influência da saúde bucal sobre a qualidade de vida de crianças préescolares. Rev Baiana Saúde Pública. 2014; 38(1):125-39.

8. Graciano MIG, Lehfeld NAS. Estudo socioeconômico: indicadores e metodologia numa abordagem contemporânea. Serv Soc \& Saúde. 2010;9(9):157-85.

9. Kleinknecht RA, Thorndike RM, McGlynn FD, Harkavy J. Factor analysis of the dental fear survey with crossvalidation. J Am Dent Assoc. 1984; 108(1):59-61.

10. Cesar J, Moraes AB, Milgrom P, Kleinknecht RA. Cross validation of a Brazilian version of the dental fear survey. Community Dent Oral Epidemiol. 1993; 21(3):148-50.

11. Lopes P, Ponciano E, Pereira A, Medeiros JA, Kleinknecht RA. Psicometria da ansiedade dentária: avaliação das características psicométricas de uma versão portuguesa do Dental Fear Survey. Rev Port Estomatol Med Dent Cir Maxilofac. 2004;45(3):133-46.

12. Ayres M, Ayres Jr M, Ayres DL, Santos AS. BioEstat 5.0: aplicações estatísticas nas áreas das ciências biomédicas [programa de computador]. Belém: Ong Mamieraua; 2007.

13. Bottan ER, Campos L, Verwiebe APS. Significado do conceito de saúde na perspectiva de escolares do ensino fundamental. Rev Bras Promoc Saúde. 2008; 21(4):240-5.

14. Costa Junior AL. Psicologia aplicada à odontopediatria: uma introdução. Estud pesqui psicol. 2002; 4(2):46-53.

15. Ramos-Jorge ML, Pordeus IA. Por que e como medir a ansiedade infantil no ambiente odontológico. Apresentação do Teste VPT modificado. JBP Rev Iberoam odontopediatr odontol Bebê. 2004; 7(37):282-90.

16. Hakeberg M, Berggren U, Carlsson SG. Prevalence of 
dental anxiety in an adult population in a major urban area in Sweden. Community Dent Oral Epidemiol. 1992; 20(2):97-101.

17. Lucas SD, Mattos FF, Melo JAC, Vasconcelos M, Abreu MHNG, Ferreira NE. Uso de metáforas para expressar a dor de dente: um estudo na área de antropologia da saúde. Ciênc saúde coletiva. 2014; 19(6):1933-42.

18. Pau A, Croucher RE, Marcenes W. Demographic and socio-economic correlates of dental pain among adults in the United Kingdom, 1998. Br Dent J. 2007; 202(9):E2; discussion 548-9..

19. Silva AM, Campos ACV, Ferreira EF, Vargas AMD. Toothaches in the Daily Lives of Brazilian Adults. Int J Environ Res Public Health. 2012; 9(8):2587-600.

20. Gonçalves CA, Vazquez FL, Ambrosano GMB, Mialhe FL, Pereira AC, Sarracini KLM, et al. Estratégias para o enfrentamento do absenteísmo em consultas odontológicas nas Unidades de Saúde da Família de um município de grande porte: uma pesquisa-ação. Ciênc saúde coletiva. 2015; 20(2):449-60.

21. Vazquez FL, Cortellazzi KL, Gonçalo CS, Bulgareli JV, Guerra LM, Tagliaferro ESP, et al. Estudo qualitativo sobre as justificativas de adolescentes para a não adesão ao tratamento odontológico. Ciênc saúde coletiva. 2015; 20(7):2147-56.

22. Barros M, Honório E. Mídia educativa ou educação midiática: os tortuosos caminhos da cidadania. Intexto. 2003; 8:1-14.

\section{CONFLITO DE INTERESSES}

Os autores declaram não haver conflitos de interesse.

\section{AUTOR PARA CORRESPONDÊNCIA}

\section{Ronald Jefferson Martins}

rojema@foa.unesp.br

Submetido em 01/11/2016

Aceito em 05/12/2016 